\title{
Surveillance du ver solitaire Echinococcus chez les coyotes et les chiens domestiques à Winnipeg, au Manitoba
}

\author{
CCK Tse ${ }^{1}$, J Bullard ${ }^{1,2,3}$, R Rusk ${ }^{1,4,5}$, D Douma6 ${ }^{6}$ PJ Plourde $1,2,5,7 *$
}

\section{Résumé}

Contexte : L'espèce Echinococcus, qui comprend E. multilocularis et E. canadensis, se compose de vers solitaires qui infectent surtout les canidés tels que les chiens, les renards et les coyotes, mais les humains sont également à risque. Chez l'humain, le ver solitaire E. multilocularis peut provoquer l'échinococcose alvéolaire, soit un trouble grave qui imite la malignité métastatique et présente un pronostic sombre. On sait que les coyotes en région rurale du Manitoba sont infectés par des espèces de ver solitaire Echinococcus, mais on ignore si les coyotes des zones périurbaines le sont également.

Objectifs : Consigner et cartographier les espèces de ver solitaire Echinococcus chez les canidés sauvages et chiens domestiques à Winnipeg, au Manitoba (Canada).

Méthodologie : On a prélevé 169 échantillons de matières fécales entre le 18 avril et le 1er juin 2018. Ils se composaient de 44 échantillons de matières fécales de chien domestique, 122 d'excréments de coyote et un d'excréments de renard, ainsi que de deux spécimens de tissu colique de coyote. On a congelé les échantillons $\left(-80^{\circ} \mathrm{C}\right)$ pendant au moins 72 heures dans le but de désactiver les ovules du ver solitaire. On a réalisé des analyses de la réaction en chaîne de la polymérase des vers solitaires $E$. multilocularis et $E$. canadensis avec les échantillons congelés.

Résultats : On a détecté des échantillons positifs pour le ver solitaire Echinococcus multilocularis dans neuf $(10,6 \%)$ des 85 emplacements, avec un échantillon positif dans un parc canin de la banlieue de Winnipeg et deux échantillons positifs dans un parc provincial populaire. Aucun échantillon de chien n'était positif pour le ver solitaire E. multilocularis; un échantillon était positif pour le ver solitaire $E$. canadensis. En revanche, neuf échantillons de coyote $(7,3 \%)$ étaient positifs pour le ver solitaire E. multilocularis et huit échantillons $(6,5 \%)$ étaient positifs pour le ver solitaire $E$. canadensis. Le seul échantillon de renard était positif pour les deux. Dans l'ensemble, six échantillons (3,6\%) étaient positifs pour les deux infections.

Conclusion : Il s'agit de la première confirmation de la présence du ver solitaire E. multilocularis dans des excréments de coyote au sein de la région métropolitaine de Winnipeg, au Manitoba. Étant donné le risque découlant de cette situation envers les chiens domestiques et la santé humaine, une surveillance périodique qui cartographie la distribution de ce ver solitaire pourrait venir appuyer le besoin de mesures de santé publique supplémentaires.

Citation proposée : Tse CCK, Bullard J, Rusk R, Douma D Plourde PJ. Surveillance du ver solitaire Echinococcus chez les coyotes et les chiens domestiques à Winnipeg, au Manitoba. Relevé des maladies transmissibles au Canada 2019;45(7/8):189-95. https://doi.org/10.14745/ccdr.45i78a01f

Mots-clés : Échinococcose alvéolaire, hydatidose, zoonoses, coyotes, renards, vers solitaires Echinococcus, chiens domestiques
Cette oeuvre est mise à la disposition selon les termes de la licence internationale Creative Commons Attribution 4.0

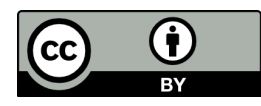

Affiliations

${ }^{1}$ Collège de médecine Max Rady, Faculté des sciences de la santé Rady, Université du Manitoba, Winnipeg, Manitoba

\section{${ }^{2}$ Département de microbiologie médicale et des maladies infectieuses, Faculté des sciences de la santé Rady, Université du Manitoba, Winnipeg, Manitoba \\ ${ }^{3}$ Laboratoire provincial Cadham, Winnipeg, Manitoba \\ ${ }^{4}$ Direction de la santé publique, Santé Manitoba, Aînés et Vie active, Winnipeg, Manitoba}

${ }^{5}$ Département des sciences de la santé communautaire, Faculté des sciences de la santé Rady, Université du Manitoba, Winnipeg, Manitoba

${ }^{6}$ Direction de la santé et du bien-être des animaux, Agriculture Manitoba, Winnipeg, Manitoba

${ }^{7}$ Programme de santé de la population et de santé publique, Office régional de la santé de Winnipeg, Winnipeg, Manitoba

*Correspondance :

pplourde@wrha.mb.ca 
L'Organisation mondiale de la Santé et l'Organisation des Nations Unies pour l'alimentation et l'agriculture ont classé l'échinococcose alvéolaire comme le troisième maladie parasitique transmise par les aliments en importance au monde (4). Vu qu'il n'existe pas de moyen rentable d'éliminer le ver solitaire E. multilocularis au cours du cycle de vie sylvatique, la surveillance est importante pour évaluer le risque chez l'humain.

\section{Comment I'humain devient infecté}

épidémiologique (figure 1). Le ver solitaire $E$. multilocularis peut provoquer une maladie grave chez l'humain, soit l'échinococcose alvéolaire $(1,2)$. Cette infection, qui se comporte comme une malignité métastatique, présente un taux de létalité élevé et nécessite un traitement chirurgical radical et un traitement anthelminthique à long terme (3). Bien qu'il s'agisse d'une infection plus bénigne chez l'humain, le ver solitaire $E$. canadensis est maintenu par un cycle de vie sylvatique qui comprend le loup, le coyote et le chien en tant qu'hôtes définitifs et le caribou, l'orignal et le wapiti en tant qu'hôtes intermédiaires. Chez l'humain, en tant que cul-desac épidémiologique, l'évolution clinique prédominante est I'hydatidose kystique principalement dans le foie, ce qui peut nécessiter une intervention chirurgicale présentant un risque $d^{\prime}$ anaphylaxie grave en cas de rupture du kyste (2). On trouve souvent ces deux espèces ensemble, puisqu'elles partagent des canidés en tant qu'hôtes définitifs. Les vers solitaires Echinococcus sont asymptomatiques et ne déclenchent pas de maladie chez les canidés, en plus d'être difficiles à détecter puisqu'ils ne mesurent que de $1 \mathrm{~mm}$ à $7 \mathrm{~mm}$.

Figure 1: Cycle de vie du ver solitaire Echinococcus multilocularis

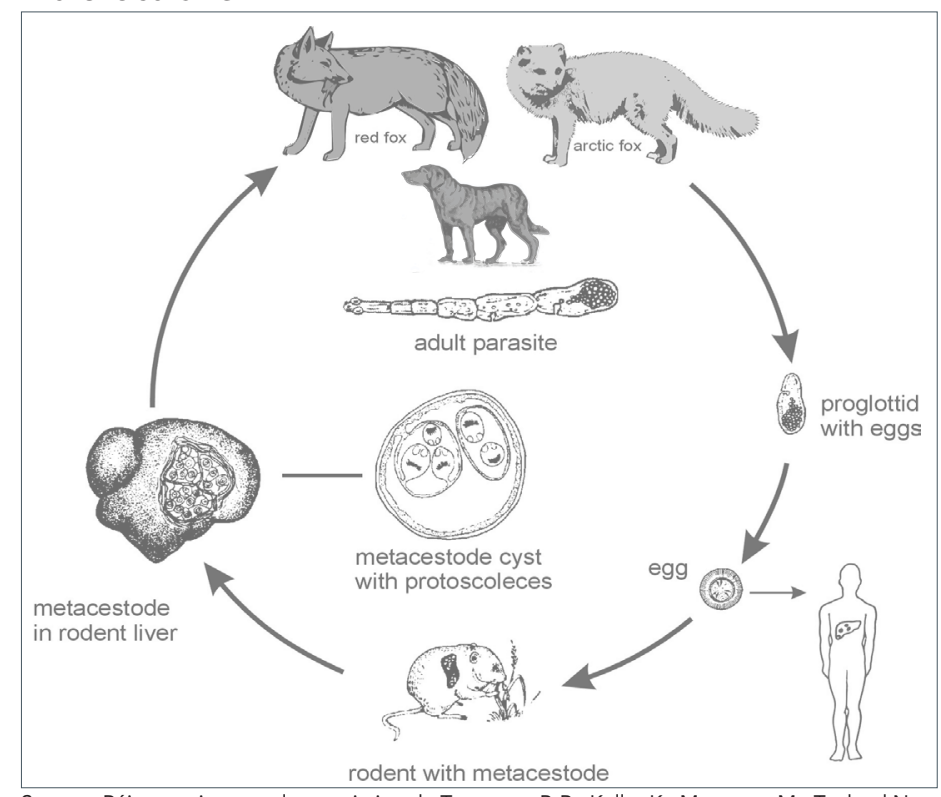

Source : Réimpression avec la permission du Torgerson P. R., Keller K., Magnotta M., Tagland N. The Global Burden of Alveolar Echnococcosis. PLoS Negl Trop Dis 2010 4(6):e722. (Figure en anglais seulement)
Les ovules du ver solitaire Echinococcus multilocularis sont très résistants et peuvent survivre et rester actifs pendant près d'un an dans des conditions favorables (5). Puisque les ovaires sont collants, ils se fixent à la fourrure des chiens, à la végétation sauvage et aux produits maraîchers cultivés dans un sol contaminé par des excréments $(2,6,7)$. En général, il y a transmission chez l'humain lorsque la main touche l'eau, le sol, la fourrure ou les objets contaminés par les excréments des hôtes, avec ensuite le transfert par inadvertance des ovules de la main à la bouche. Après l'ingestion humaine d'ovules du ver solitaire E. multilocularis, il y a une longue période de latence clinique, soit cinq à quinze ans. Les premiers symptômes comprennent de la douleur abdominale, qui est suivie de jaunisse et, au bout du compte, de dysfonctionnement hépatique grave $(2,8)$. On méprend souvent l'échinococcose alvéolaire pour une croissance néoplasique, et ce, en raison de sa tendance à I'infiltration organique répandue avec métastases (2). Le ver solitaire E. multilocularis peut également imiter d'autres maladies telles que le carcinome hépatique, la cirrhose et la tuberculose, ce qui peut mener à des épreuves diagnostiques inadéquates et un retard de traitement $(3,9,10)$. On confirme le diagnostic par une combinaison de résultats cliniques, de données épidémiologiques, d'imagerie, d'histopathologie ou de détection d'acide nucléique et de sérologie (8). Le traitement recommandé est la résection chirurgicale radicale de la lésion parasitaire qui, lors des premiers stades, peut mener à une guérison complète; cependant, la résection chirurgicale peut parfois être incomplète pour cause de propagation diffuse ou non détectée du parasite $(2,8)$. À ce titre, on recommande une chimiothérapie anthelminthique postopératoire pendant au moins deux ans, suivie d'au moins dix ans de surveillance étroite (8). Sans traitement, la mortalité est de $90 \%$ dans les dix années qui suivent l'apparition des symptômes (11). On a démontré qu'un diagnostic précoce et un traitement approprié améliorent les chances de survie (12).

\section{Le ver solitaire Echinococcus multilocularis au Canada}

On note des vers solitaires Echinococcus chez les canidés du Canada depuis nombre d'années. Une étude sur la prévalence du ver solitaire E. multilocularis chez les espèces de canidés du Manitoba, réalisée il y a 40 ans au parc national du 
Mont-Riding, a permis de constater que presque le quart des coyotes échantillonnés étaient infectés (13). Plus récemment, en 2009, on a découvert une souche européenne du ver solitaire E. multilocularis au centre de la Colombie-Britannique chez un chien domestique sans antécédent de déplacement à l'extérieur du Canada; d'autres études laissent entendre l'établissement possible de cette nouvelle souche dans la faune locale $(14,15)$. Il pourrait y avoir des preuves d'une souche semblable au sein de la faune de la Saskatchewan (16). Des enquêtes à Calgary et Edmonton publiées en 2012 ont permis de déterminer la présence du ver solitaire E. multilocularis au sein de la population sauvage de canidés de ces villes (1). De plus, en Ontario, des détections récentes d'échinococcose alvéolaire chez plusieurs chiens domestiques sans antécédent de déplacement à l'extérieur de l'Ontario laissent entendre que le ver solitaire E. multilocularis pourrait aussi avoir établi un foyer dans le sud-ouest de l'Ontario $(17,18)$.

Les vers solitaires Echinococcus et les cas d'échinococcose alvéolaire humaine ont d'abord été signalés dans les années 1930 au Manitoba et en Alberta (19). Plus récemment, en novembre 2017, on a diagnostiqué une infection disséminée d'échinococcose alvéolaire chez un patient pédiatrique du Manitoba (communication personnelle, 23 novembre 2017, Dr Sergio Fanella). Dans le même ordre d'idées, en 2018, on a détecté plusieurs cas humains d'échinococcose alvéolaire en Alberta (communication personnelle, 5 juin 2018, Dr Stan Houston).

Vu la hausse de l'urbanisation et des observations de coyotes dans les zones urbaines et suburbaines, le ver solitaire E. multilocularis peut se rapprocher des chiens domestiques et humains $(20,21)$. Étant donné ces cas humains récents et les observations de coyotes à Winnipeg et aux alentours de la ville (20), on a procédé à une enquête sur les canidés sauvages pour étudier la présence d'espèces de vers solitaires Echinococcus.

Cette étude a pour but de déterminer l'étendue des ovaires d'espèces d'Echinococcus, en particulier le ver solitaire E. multilocularis, dans les excréments de coyote, renard et chien domestique trouvés dans la région métropolitaine qui entoure Winnipeg, ainsi que de créer des cartes géographiques visant à déterminer les zones à risque.

\section{Méthodologie}

\section{Lieux de prélèvement}

On a défini la région métropolitaine de Winnipeg (RMW) en tant que région métropolitaine de recensement 602 conformément au profil du recensement de 2016 de Statistique Canada (22). Elle se compose du milieu urbain, délimité par l'autoroute périphérique circonférentielle qui entoure Winnipeg et la zone périurbaine à l'extérieur de l'autoroute périphérique et au sein de la RMW. On a identifié 85 lieux de prélèvement couvrant une grande zone de la RMW, y compris des zones fréquentées par des humains et chiens domestiques, ainsi que des zones avec des observations connues de coyotes.

\section{Prélèvement des échantillons}

On a prélevé des échantillons fécaux entre le 18 avril et le $1^{\text {er }}$ juin 2018. On a prélevé les échantillons de chiens domestiques d'une manière qui diffère de celle pour les autres échantillons de canidés. On a prélevé les échantillons de chiens dans des parcs canins et on en a reçu directement de propriétaires de chiens. On a offert deux points de remise aux bénévoles qui remettaient des échantillons de matière fécale provenant de chiens domestiques.

On a surtout prélevé des échantillons de canidés sauvages (principalement des coyotes) en conduisant le long de routes éloignées près de l'habitat des coyotes conformément aux observations locales connues de coyotes. On a identifié les échantillons de coyote à l'aide de caractéristiques de l'échantillon d'excréments, dont la forme, la texture, la présence de poils ou d'os appartenant à de petits animaux, ainsi que de caractéristiques à l'appui telles que des traces de coyotes, des tanières de coyotes à proximité et des observations antérieures (20). Dans le même ordre d'idées, l'agent de conservation local a différencié l'échantillon d'excréments de renard par sa petite taille et le fait qu'il y a eu des observations de renards aux alentours.

On a mis les échantillons fécaux dans des sacs de plastique individuels, scellés et étiquetés avec la date du prélèvement et l'endroit du prélèvement [coordonnées du système mondial de localisation (GPS), code postal ou adresse]. Pour les échantillons de chien domestique, on a également inscrit le nom et le numéro de téléphone du propriétaire du chien. Des agents locaux de conservation de la faune qui ont retiré le côlon de deux animaux uniques ont remis deux échantillons de côlon prélevés sur des carcasses de coyotes. On a entreposé les échantillons à $-80^{\circ} \mathrm{C}$ pendant au moins 72 heures dans le but de désactiver les ovules du ver solitaire E. multilocularis. Les échantillons coliques ont été grattés sur la surface muqueuse pour en retirer les matières fécales.

\section{Épreuves de laboratoire}

On a entreposé et transporté les matières fécales dans des contenants fécaux stériles conservés sur de la glace et envoyés par livraison le jour même au laboratoire de référence (IDEXX Laboratories, Inc.) à Markham, en Ontario. On a traité les échantillons fécaux immédiatement à leur arrivée, en plus de réaliser une extraction de l'acide nucléique total au moyen $d^{\prime}$ un processeur de particules magnétiques KingFisher ${ }^{\mathrm{MC}}$ Flex 
On a détecté des échantillons positifs pour le ver solitaire Echinococcus multilocularis dans neuf $(10,6 \%)$ des

85 emplacements, avec un échantillon positif dans un parc canin de la banlieue de Winnipeg et deux échantillons positifs au parc provincial Birds Hill. Les autres échantillons semblent mieux répandus parmi les milieux plus ruraux de la RMW. La figure 2 indique les lieux de prélèvement des échantillons, ainsi que l'emplacement des échantillons positifs de ver solitaire E. multilocularis.

Figure 2 : Carte de la distribution du prélèvement des échantillons de canidés dans la région métropolitaine de Winnipeg, qui indique les échantillons positifs pour le ver solitaire Echinococcus multilocularis

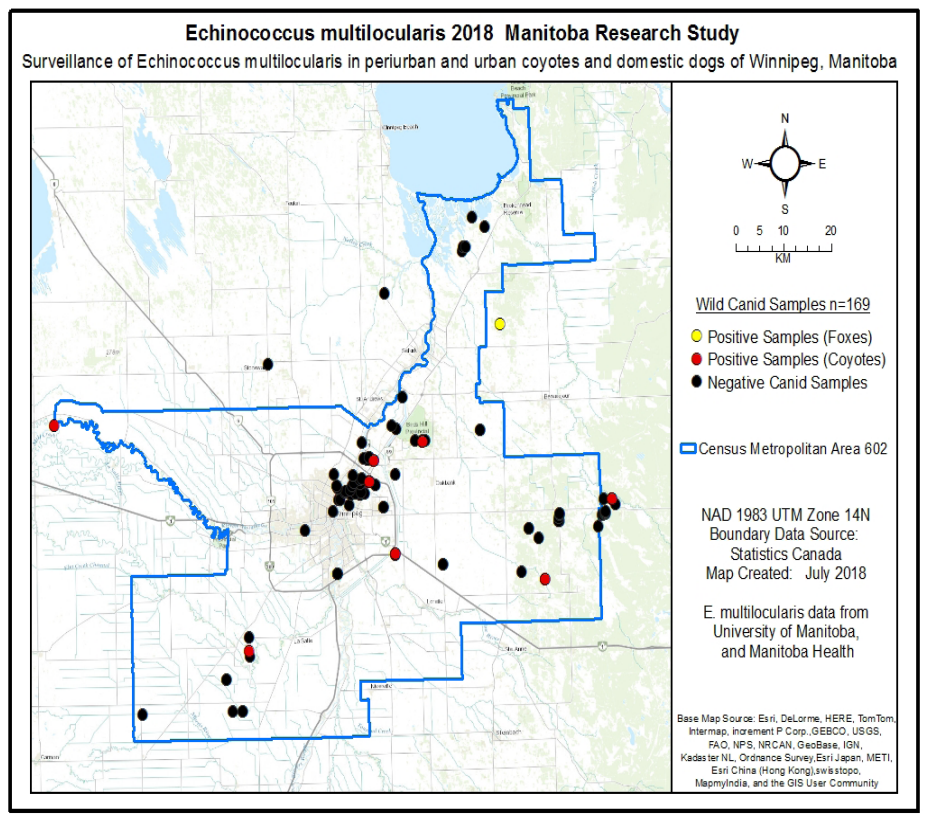

Un point rouge indique un échantillon positif pour le ver solitaire $E$. multilocularis avec les excréments ou le tissu de coyote, un point jaune indique un échantillon positif pour le ver solitaire E. multilocularis avec les excréments de renard et un point noir indique un échantillon de canidé négatif pour le ver solitaire $E$. multilocularis. Le tracé bleu représente la région métropolitaine de recensement de Winnipeg - avec la permission de Statistique Canada (22) (Figure en anglais seulement)

Parmi les échantillons envoyés aux fins de traitement moléculaire, dix $(5,9 \%)$ étaient positifs pour le ver solitaire $E$. canadensis et dix $(5,9 \%)$ étaient positifs pour le ver solitaire E. multilocularis, avec six $(3,6 \%)$ coinfections chez cinq coyotes et un renard. On a trouvé le ver solitaire E. multilocularis dans $7,3 \%$ des échantillons de coyote, dans $0 \%$ des échantillons de canidé domestique et dans l'échantillon unique de renard. On a trouvé le ver solitaire E. canadensis dans $6,5 \%$ des échantillons de coyote, dans 2,3\% des échantillons de chien domestique et dans l'échantillon unique de renard (tableau 1). d'animaux uniques prélevés directement des propriétaires des chiens (on a prélevé les 10 autres échantillons dans des parcs canins et ils ne proviennent pas nécessairement d'animaux uniques) et un échantillon d'excrément de renard.
On a prélevé un total de 169 échantillons, qui comprenaient 122 échantillons d'excréments de coyote, deux échantillons de tissu colique de coyote, 44 échantillons fécaux de chien domestique, parmi lesquels 34 échantillons provenaient 
Tableau 1 : Nombre et pourcentage d'échantillons de canidé infectés par les vers solitaires Echinococcus dans les zones urbaines et périurbaines de Winnipeg, 2018

\begin{tabular}{|c|c|c|c|c|c|c|}
\hline \multirow{2}{*}{$\begin{array}{l}\text { La source } \\
\text { d'échantillons } \\
\text { de selles }\end{array}$} & \multicolumn{3}{|c|}{ Echinococcus multilocularis } & \multicolumn{3}{|c|}{ Echinococcus canadensis } \\
\hline & $\begin{array}{c}\text { Nombre } \\
\text { d'échantillon }\end{array}$ & $\begin{array}{c}\text { Nombre de } \\
\text { selles positives }\end{array}$ & $\begin{array}{c}\% \text { d'échantillons } \\
\text { positifs }\end{array}$ & $\begin{array}{c}\text { Nombre } \\
\text { d'échantillons }\end{array}$ & $\begin{array}{l}\text { Nombre de } \\
\text { selles positives }\end{array}$ & $\begin{array}{c}\% \text { d'échantillons } \\
\text { positifs }\end{array}$ \\
\hline $\begin{array}{l}\text { Chien } \\
\text { domestique }^{\text {a }}\end{array}$ & 44 & 0 & 0 & 44 & 1 & 2,3 \\
\hline Coyote $^{b}$ & 124 & 9 & 7,3 & 124 & 8 & 6,5 \\
\hline Renard & 1 & 1 & 100 & 1 & 1 & 100 \\
\hline Total & 169 & 10 & 5,9 & 169 & 10 & 5,9 \\
\hline
\end{tabular}

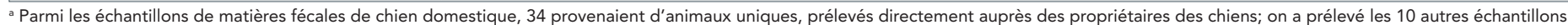
dans des parcs canins et ils ne proviennent pas nécessairement d'animaux uniques

${ }^{b}$ Les 124 échantillons d'excréments de coyote ne proviennent pas nécessairement d'animaux uniques; par conséquent, plusieurs échantillons pourraient provenir du même coyote

\section{Discussion}

Il s'agit de la première confirmation de la présence du ver solitaire E. multilocularis à la fois dans les zones urbaines et périurbaines de Winnipeg, au Manitoba. La distribution du ver solitaire $E$. multilocularis chez le coyote semble répandue, sans point névralgique. Bien qu'on ait identifié le ver solitaire E. multilocularis dans un parc provincial récréatif populaire et un parc canin, aucun échantillon de chien domestique n'était positif pour ce ver solitaire. On a trouvé un seul cas du ver solitaire E. canadensis beaucoup plus bénin dans un échantillon de chien domestique.

Les limites de la présente étude comprennent la courte durée de la surveillance, l'échantillonnage répété possible provenant des mêmes canidés et l'usage d'un essai moléculaire qui est encore en cours de révision par les pairs (23). On n'a pas prélevé de caractéristique des chiens domestiques, telle qu'à savoir s'il s'agissait de chiens d'extérieur ou s'ils consommaient des rongeurs. On a ciblé la méthode d'échantillonnage d'excréments de canidé sauvage et, par conséquent, elle ne reflète pas un prélèvement uniformisé à l'échelle de la région. II faudrait une taille d'échantillon plus grande et un délai de surveillance plus long de façon à mieux cartographier l'étendue de l'infection de canidés par le ver solitaire $E$. multilocularis dans la région métropolitaine de Winnipeg.

\section{Conséquences et prochaines étapes}

Nos constatations indiquent un risque d'exposition des humains et chiens domestiques aux excréments de coyote infectés par le ver solitaire E. multilocularis dans les zones urbaines et périurbaines de Winnipeg.

On ne sait pas exactement la manière à laquelle l'exposition humaine au parasite entraîne une infection provoquant une échinococcose alvéolaire. En 2001, on a publié une étude quinquennale en Suisse qui indique un taux de séropositivité humaine élevé pour le ver solitaire E. multilocularis, et ce, sans hausse du taux d'attaque. Une explication était une immunité accrue potentielle au sein de cette population (25). Cependant, un examen de 2007 a permis de découvrir une hausse importante de l'incidence d'échinococcose alvéolaire en Suisse après 2000, qui était précédée par une augmentation de cette infection dans la population locale de renards 10 à 15 ans plus tôt (26). On était d'avis que cette situation reflétait la longue période de latence clinique de l'échinococcose alvéolaire. Une étude plus longue est en cours afin de déterminer si la prévalence élevée du ver solitaire E. multilocularis dans l'environnement est associée à une incidence accrue d'échinococcose alvéolaire chez l'humain (25).

En fonction des preuves de ver solitaire E. multilocularis dans l'environnement dans plusieurs provinces et des cas humains récents en Alberta et au Manitoba, d'autres études sont indiquées. Dans le but de mieux caractériser la prévalence et la distribution géographique, on recommande de faire une recherche avec un plus grand nombre de lieux et d'échantillons, une analyse spatiale et des capacités de diagnostic moléculaire au sein de la province. Si la situation le justifie, on pourrait considérer une étude de séropositivité humaine du ver solitaire E. multilocularis dans les régions touchées.

Cet enjeu émergent peut tout spécialement relever d'une approche "Un monde, une santé » (27) qui comprend des médecins, vétérinaires et experts de la faune. L'Ontario oblige la déclaration de l'infection par le ver solitaire $E$. multilocularis chez le chien domestique et l'humain dans l'optique de surveiller l'étendue de l'infection $(28,29)$. Bien que ce ne soit pas obligatoire ailleurs à l'heure actuelle, il serait utile de faire un compte rendu de l'enquête sur les cas humains d'échinococcose alvéolaire, y compris les antécédents de déplacement (pour confirmer la maladie acquise sur le plan local par rapport à celle importée de pays étrangers) et de fournir de l'information sur la voie d'exposition potentielle aux ovules du ver solitaire E. multilocularis. La sensibilisation du public à propos des mesures préventives visant à prévenir les infections chez le chien domestique et l'humain pourrait être indiquée. 


\section{Conclusion}

On documente le ver solitaire Echinococcus multilocularis au Canada depuis plusieurs décennies au sein de la population de canidés sauvages, mais les cas humains se sont avérés extrêmement rares. Au cours des 20 dernières années, on a observé que les canidés sauvages ont tendance à migrer plus près des populations urbaines, ainsi que plusieurs cas humains récents d'échinococcose alvéolaire causés par le ver solitaire E. multilocularis. Bien que notre étude n'ait pas constaté de contamination croisée entre les coyotes et les chiens à Winnipeg, le risque d'exposition au ver solitaire E. multilocularis dans des milieux urbains et périurbains laisse entendre qu'une sensibilisation accrue et d'autres études pourraient aider les cliniciens de première ligne et les responsables de la santé publique à être à l'affût du risque et le surveiller.

\section{Déclaration des auteurs}

C. C. K. T. - Conception du projet, recherches documentaires, méthodologie, échantillonnage sur le terrain, essais en laboratoire, analyse des données, rédaction (première ébauche, révision et mise en forme)

J. B. - Conception du projet, méthodologie, essais en laboratoire " en nature », assistance logicielle, analyse des données, rédaction (révision et mise en forme)

R. R. - Conception du projet, méthodologie, assistance logicielle, analyse des données spatiales, rédaction (révision et mise en forme)

D. D. - Conception du projet, méthodologie, essais en laboratoire "en nature ", analyse des données, rédaction (révision et mise en forme)

P. J. P. - Conception du projet, recherches documentaires, méthodologie, soutien de l'échantillonnage sur le terrain, analyse des données, rédaction (révision et mise en forme)

\section{Conflit d'intérêts}

Aucun.

\section{Remerciements}

Les auteurs souhaitent remercier le Laboratoire provincial Cadham et le département de parasitologie pour le soutien de laboratoire, de même que D. Berezanski et la Direction de la faune et de la pêche (ministère du Développement durable du Manitoba) pour l'aide apportée avec le prélèvement des échantillons et la sensibilisation des bénévoles vis-à-vis des coyotes. Un grand merci à C. Swiderek, au Kilcona Dog Park Club et aux bénévoles pour leur aide lors du prélèvement des échantillons. Nous souhaitons remercier T. Madill et G. Houghton pour leur aide lors du prélèvement et du traitement des échantillons, ainsi qu'IDEXX Laboratories Inc. pour l'analyse moléculaire des échantillons.
Les résultats et conclusions sont ceux des auteurs il ne faut pas conclure à l'aval officiel de Santé Manitoba, d'Aînés et Vie active ou d'Agriculture Manitoba.

\section{Financement}

La présente étude a reçu de l'appui pour C. C. K. Tse, un étudiant d'été qui a réalisé un projet de recherche de deuxième année de médecine d'avril à août 2018.

\section{Références}

1. Catalano S, Lejeune M, Liccioli S, Verocai GG, Gesy KM, Jenkins EJ, Kutz SJ, Fuentealba C, Duignan PJ, Massolo A. Echinococcus multilocularis in urban coyotes, Alberta, Canada. Emerg Infect Dis 2012 Oct;18(10):1625-8. DOI PubMed

2. Eckert J, Deplazes P. Biological, epidemiological, and clinical aspects of echinococcosis, a zoonosis of increasing concern. Clin Microbiol Rev 2004 Jan;17(1):107-35. DOI PubMed

3. Moro P, Schantz PM. Echinococcosis: a review. Int J Infect Dis 2009 Mar;13(2):125-33. DOI PubMed

4. Food and Agriculture Organization of the United Nations and World Health Organization. Multicriteria-Based Ranking for Risk Management of Food-Borne Parasites. Geneva (Switzerland); WHO/FAO: 2014. www.fao.org/3/a-i3649e. pdf\%5Cnfiles/540/a-i3649e.pdf

5. Veit P, Bilger B, Schad V, Schäfer J, Frank W, Lucius R. Influence of environmental factors on the infectivity of Echinococcus multilocularis eggs. Parasitology 1995 Jan;110(Pt 1):79-86. DOl PubMed

6. Jenkins EJ, Castrodale LJ, de Rosemond SJ, Dixon BR Elmore SA, Gesy KM, Hoberg EP, Polley L, Schurer JM, Simard M, Thompson RC. Tradition and transition: parasitic zoonoses of people and animals in Alaska, northern Canada, and Greenland. Adv Parasitol 2013;82:33-204. DOI PubMed

7. Hildreth MB, Sriram S, Gottstein B, Wilson M, Schantz PM. Failure to identify alveolar echinococcosis in trappers from South Dakota in spite of high prevalence of Echinococcus multilocularis in wild canids. J Parasitol 2000 Feb;86(1):75-7. DOI PubMed

8. Brunetti E, Kern P, Vuitton DA; Writing Panel for the WHO-IWGE. Expert consensus for the diagnosis and treatment of cystic and alveolar echinococcosis in humans. Acta Trop 2010 Apr;114(1):1-16. DOI PubMed

9. Wilson JF, Rausch RL. Alveolar hydatid disease. A review of clinical features of 33 indigenous cases of Echinococcus multilocularis infection in Alaskan Eskimos. Am J Trop Med Hyg 1980 Nov;29(6):1340-55. DOI PubMed

10. Caire Nail L, Rodríguez Reimundes E, Weibel Galluzzo C, Lebowitz D, Ibrahim YL, Lobrinus JA, Chappuis F. Disseminated alveolar echinococcosis resembling metastatic malignancy: a case report. J Med Case Reports 2017 Apr;11(1):113. DOI PubMed 
11. Ammann RW, Eckert J. Cestodes. Echinococcus. Gastroenterol Clin North Am 1996 Sep;25(3):655-89. DOI PubMed

12. Torgerson PR, Schweiger A, Deplazes P, Pohar M, Reichen J, Ammann RW, Tarr PE, Halkik N, Müllhaupt B. Alveolar echinococcosis: from a deadly disease to a well-controlled infection. Relative survival and economic analysis in Switzerland over the last 35 years. J Hepatol 2008 Jul;49(1):72-7. DOl PubMed

13. James E, Boyd W. Echinococcus Alveolaris: (With the Report of a Case). Can Med Assoc J 1937 Apr;36(4):354-6. PubMed

14. Samuel WM, Ramalingam S, Carbyn LN. Helminths in coyotes (Canis latrans Say), wolves (Canis lupus L.), and red foxes (Vulpes vulpes L.) of southwestern Manitoba. Can J Zool 1978 Dec;56(12):2614-7. DOI PubMed

15. Government of Manitoba. Living with Wildlife in Manitoba (Accédé 2018-08-03). www.gov.mb.ca/sd/wildlife/problem_ wildlife/coyote.html

16. Deplazes P, Hegglin D, Gloor S, Romig T. Wilderness in the city: the urbanization of Echinococcus multilocularis. Trends Parasitol 2004 Feb;20(2):77-84. DOI PubMed

17. Skelding A, Brooks A, Stalker M, Mercer N, deVilla E, Gottstein B, Peregrine AS. Hepatic alveolar hydatid disease (Echinococcus multilocularis) in a boxer dog from southern Ontario. Can Vet J 2014;55:551-553. PubMed

18. Trotz-Williams LA, Mercer NJ, Walters JM, Wallace D, Gottstein B, Osterman-Lind E, Boggild AK, Peregrine AS. Public health follow-up of suspected exposure to Echinococcus multilocularis in Southwestern Ontario. Zoonoses and Public Health 2017;64:460-467. DOI PubMed

19. Jenkins EJ, Peregrine AS, Hill JE, Somers C, Gesy K, Barnes B, Gottstein B, Polley L. Detection of European strain of Echinococcus multilocularis in North America. Emerg Infect Dis 2012 Jun;18(6):1010-2. DOI PubMed

20. Gesy K, Hill JE, Schwantje H, Liccioli S, Jenkins EJ. Establishment of a European-type strain of Echinococcus multilocularis in Canadian wildlife. Parasitology 2013 Aug;140(9):1133-7. DOl PubMed

21. Gesy KM, Jenkins EJ. Introduced and Native Haplotypes of Echinococcus multilocularis in Wildlife in Saskatchewan, Canada. J Wildl Dis 2015 Jul;51(3):743-8. DOI PubMed
22. Statistique Canada. 2017. Winnipeg [Région métropolitaine de recensement], Manitoba et Manitoba [Province]. Profil du recensement, Recensement 2016. Statistique Canada No. 98-316-X2016001 au catalogue. Ottawa (ON): Stats Can; le 29 novembre 2017 (Accédé 2018-07-25). www12. statcan.gc.ca/census-recensement/2016/dp-pd/prof/index. cfm?Lang $=F$

23. Sindern N, Suchodolski JS, Leutenegger CM, Mehdizadeh Gohari I, Prescott JF, Proksch AL, Mueller RS, Busch K, Unterer S. Prevalence of Clostridium perfringens netE and netF toxin genes in the feces of dogs with acute hemorrhagic diarrhea syndrome. J Vet Intern Med 2019 Jan;33(1):100-5. DOI PubMed

24. Isaksson $M$, Hagström $\AA$, Armua-Fernandez MT, Wahlström H, Ågren EO, Miller A, Holmberg A, Lukacs M, Casulli A, Deplazes $P$, Juremalm M. A semi-automated magnetic capture probe based DNA extraction and real-time PCR method applied in the Swedish surveillance of Echinococcus multilocularis in red fox (Vulpes vulpes) faecal samples. Parasit Vectors 2014 Dec;7:583. DOI PubMed

25. Gottstein B, Saucy F, Deplazes P, Reichen J, Demierre G, Busato A, Zuercher C, Pugin P. Is high prevalence of Echinococcus multilocularis in wild and domestic animals associated with disease incidence in humans? Emerg Infect Dis 2001 May-Jun;7(3):408-12. DOI PubMed

26. Schweiger A, Ammann RW, Candinas D, Clavien PA, Eckert J, Gottstein B, Halkic N, Muellhaupt B, Prinz BM, Reichen J, Tarr PE, Torgerson PR, Deplazes P. Human alveolar echinococcosis after fox population increase, Switzerland. Emerg Infect Dis 2007 Jun;13(6):878-82. DOl PubMed

27. One Health Initiative. About the One Health Initiative. (Accédé 2018-08-09). www.onehealthinitiative.com/about. php

28. Ontario Ministry of Health and Long-Term Care. Management of Echinococcus multilocularis Infections in Animals Guideline, 2018 (Accédé 2018-12-18). http://health. gov.on.ca/en/pro/programs/publichealth/oph_standards/ docs/protocols_guidelines/Management_of_EM_Infections_ in_Animals_2018.pdf

29. Gouvernement de I'Ontario. R.R.O. 1990, Règl. 557: MALADIES TRANSMISSIBLES - DISPOSITION GÉNÉRALES. Toronto (ON): Gouvernement de I'Ontario; 2017 (Accédé 2018-07-25). www.ontario.ca/laws/regulation/900557/v5 